\title{
Intelligent Wireless Sensors with Application to the Identification of Structural Modal Parameters and Steel Cable Forces: From the Lab to the Field
}

\author{
Y. Lei, ${ }^{1}$ W. A. Shen, ${ }^{1}$ Y. Song, ${ }^{1}$ and Y. Wang ${ }^{2}$ \\ ${ }^{1}$ Department of Civil Engineering, Xiamen University, Xiamen, Fujian 361005, China \\ ${ }^{2}$ School of Civil and Environmental Engineering, Georgia Institute of Technology, Atlanta, GA 30332, USA
}

Correspondence should be addressed to Y. Lei, ylei@xmu.edu.cn

Received 3 October 2009; Revised 1 February 2010; Accepted 6 May 2010

Academic Editor: Piervincenzo Rizzo

Copyright ( 2010 Y. Lei et al. This is an open access article distributed under the Creative Commons Attribution License, which permits unrestricted use, distribution, and reproduction in any medium, provided the original work is properly cited.

\begin{abstract}
Wireless sensing systems have been proposed for structural heath monitoring in recent years. While wireless sensors are cost-competitive compared to tethered monitoring systems, their significant merit also lies in their embedded computational capabilities. In this paper, performance of the two embedded engineering algorithms, namely the fast Fourier transform and peak-picking algorithm implemented in the wireless sensing nodes codeveloped at Stanford University and the University of Michigan is investigated through laboratory and field experimental studies. Furthermore, the wireless sensor network embedded with the engineering algorithms is adopted for the identification of structural modal parameters and forces in steel bridge cables. Identification results by the embedded algorithms in the intelligent wireless sensors are compared with those obtained by conventional offline analysis of the measured time-history data. Such a comparison serves to validate the effectiveness of the intelligent wireless sensor network. In addition, it is shown that self-interrogation of measurement data based upon the two embedded algorithms in wireless sensor nodes greatly reduces the amount of data to be transmitted by the wireless sensing network. Thus, the intelligent wireless sensors offer scalable network solutions that are power-efficient for the health monitoring of civil infrastructures.
\end{abstract}

\section{Introduction}

Conventional structural monitoring systems are wire-based systems, which require extensive lengths of cables to transmit recorded data from multiple sensors to a centralized data repository. Installation and maintenance of such wired systems for the structural health monitoring (SHM) of civil infrastructures, including high-rise buildings and long-span bridges, is time consuming and expensive. With the advances in wireless communication and microelectronics, wireless monitoring systems have been proposed to eradicate the extensive lengths of wires associated with tethered systems [ 1 , $2]$. In recent years, some academic and commercial wireless monitoring systems have been proposed $[2,3]$. Among them, the academic wireless sensing prototype codeveloped at Stanford University and the University of Michigan has received great attention as it emphasizes the design of a sophisticated computational core that can process measurement data at the wireless sensor level [4-6]. The data acquisition capabilities of this wireless sensing system have been validated through both laboratory and field experimental studies [7-9].

While wireless monitoring systems offer an economical and feasible technique for data acquisition in SHM, transmitting the lengthy vibration data to the base station can be undesirable due to the limited bandwidth of the wireless communication. Sending a tremendous amount of raw data to a central server consumes excessive battery energy as communication is responsible for more than $80 \%$ of energy consumption at full operation mode in ultra-lowpower platforms [10]. Overall, wireless data transmission consumes more power than data processing; therefore, one of the efficient strategies for overcoming these difficulties is to embed some data processing and analysis algorithms in the wireless sensor's microprocessor. The embedded algorithms enable wireless sensors to autonomously analyze data, which grants these devices with certain level of intelligence. In addition, the wireless sensors only need to transmit analysis results, which typically have only a fraction of the size of 
raw time history data. As a result, the limitations in terms of energy consumption and network bandwidth can be mitigated. This capability is particularly attractive within the context of SHM $[1,2,5]$. So far, a number of data processing and analysis algorithms have been implemented in the academic wireless sensing nodes as discussed by Lynch et al. $[4,5]$, but their performance still needs to be validated by further tests on different structures.

In this paper, two embedded engineering algorithms implemented in the wireless sensor, including the fast Fourier transform (FFT) and peak-picking (PP) algorithms, are investigated through laboratory experiments on a three-story structural model as well as through field studies on a steel arch bridge under ambient vibration. Focus is placed upon validating the performance of the two embedded algorithms and their effectiveness in reducing energy consumptions. Online data processing and analysis results by the two embedded algorithms are compared to those obtained by offline analysis of the measured raw time-history data. This side-by-side comparison validates the accuracy of the embedded algorithms. Finally, the two embedded algorithms are employed for identifying the modal parameters of the laboratory building model and the steel arch bridge as well as for estimating cable forces in the steel arch bridge based upon the vibration data.

\section{Wireless Sensing Units}

The overall hardware of the academic wireless sensing prototype codeveloped at Stanford University and the University of Michigan [2,3] mainly consists of three functional modules: (1) sensing interface, (2) computational core, and (3) wireless communication module, as shown in Figure 1. In the computation core, a low-cost, low-power 8-bit Atmel AVR microcontroller (ATmega 128) is selected to coordinate the hardware components of the wireless unit. The microcontroller, together with ample internal and external memories, provides the capability of onboard data interrogation at the sensor level. Detailed descriptions on the key parameters of ATmega128 and the computational core can be found in Wang [3] and Wang et al. [10]. The wireless sensing unit is particularly designed to balance low power consumption while supporting high-data transfer rate and long communication range typically required for civil structural applications [10].

As shown in Figure 2, the embedded software is structured based upon a multilayer architecture $[2,3,5]$. At the lower layer of the embedded software architecture is the device driver layer which directly manages the wireless sensor hardware. Software, including the FFT and PP schemes, that processes and analyzes sensor data resides on the upper application layer of the software architecture.

\section{Implementation of the FFT and PP Algorithms}

The fast Fourier transform (FFT) is an efficient algorithm to transform time history data into frequency domain. It also serves as the foundation for further frequency domain structural identification and damage detection analysis, for example, the peak-picking (PP) algorithm is based upon the results of FFT. In the wireless sensing node, the FFT algorithm is implemented in floating point numbers and is computed based upon the following equation:

$$
F_{x}(k)=\sum_{n=1}^{N} x(n) \exp \left(-\frac{i 2 \pi(k-1)(n-1)}{N}\right), \quad 1 \leq k \leq N
$$

where $N=4096$. The FFT can be executed onboard, as soon as the sensor data is collected. When the interest is focused upon the first few vibration modes of a civil structure, only the FFT results within specific frequency range need to be transmitted from each sensing node to the central server [10]. In addition, the 4096-point complex-valued Fourier spectrum can also be used for further onboard engineering analysis by the wireless nodes, and only the analysis results are transmitted to the server.

The peak-picking (PP) algorithm is a simple approach for estimating the modal properties of a structure. The peakpicking analysis can be based upon output vibration data only, that is, without requiring input measurement, which makes it commonly used in civil engineering. If a structure is lighted and damped with well-separated modes, the natural frequencies and operational mode shapes of the structure can be estimated with the PP approach, using the frequency response functions (FRFs). Assuming a white noise excitation, the FRF can be considered equivalent to the Fourier spectrum of the response data, which is obtained through the fast Fourier transform (FFT). The implementation of the PP algorithm embedded in the wireless sensing nodes is described as follows:

(1) The time history data collected at each sensing node is converted into a FRF by executing the embedded FFT algorithm.

(2) The average value of the FRF over the frequency span is calculated as $\mu$. Then a threshold value, defined as $\beta=n \mu$, is selected, where $n$ is a scaling factor assigned by the user. The threshold value is used to eliminate those small spurious local peaks in the FRF, which exist due to sensor noise and environmental influences. A carefully assigned threshold value assists in eliminating small spurious peaks, while allowing the algorithm to pick up the nonspurious peaks in the frequency spectrum.

(3) Based upon the total number of peaks, $p$, that are to be identified, each sensing node picks the $p$ peaks by scanning for frequencies at which the absolute values of the corresponding FRF are not only larger than the threshold value $\beta$, but also larger than the surrounding points in the FRF spectrum as

$$
\begin{array}{ll}
\left|F_{x}(k)\right|>\left|F_{x}(k-1)\right|, & \left|F_{x}(k-2)\right|, \\
\left|F_{x}(k)\right|>\left|F_{x}(k+1)\right|, & \left|F_{x}(k+2)\right|,
\end{array}
$$

in which $\left|F_{x}(k)\right|$ is the absolute value of the selected peak in the spectrum. In this approach, it is assumed that the 


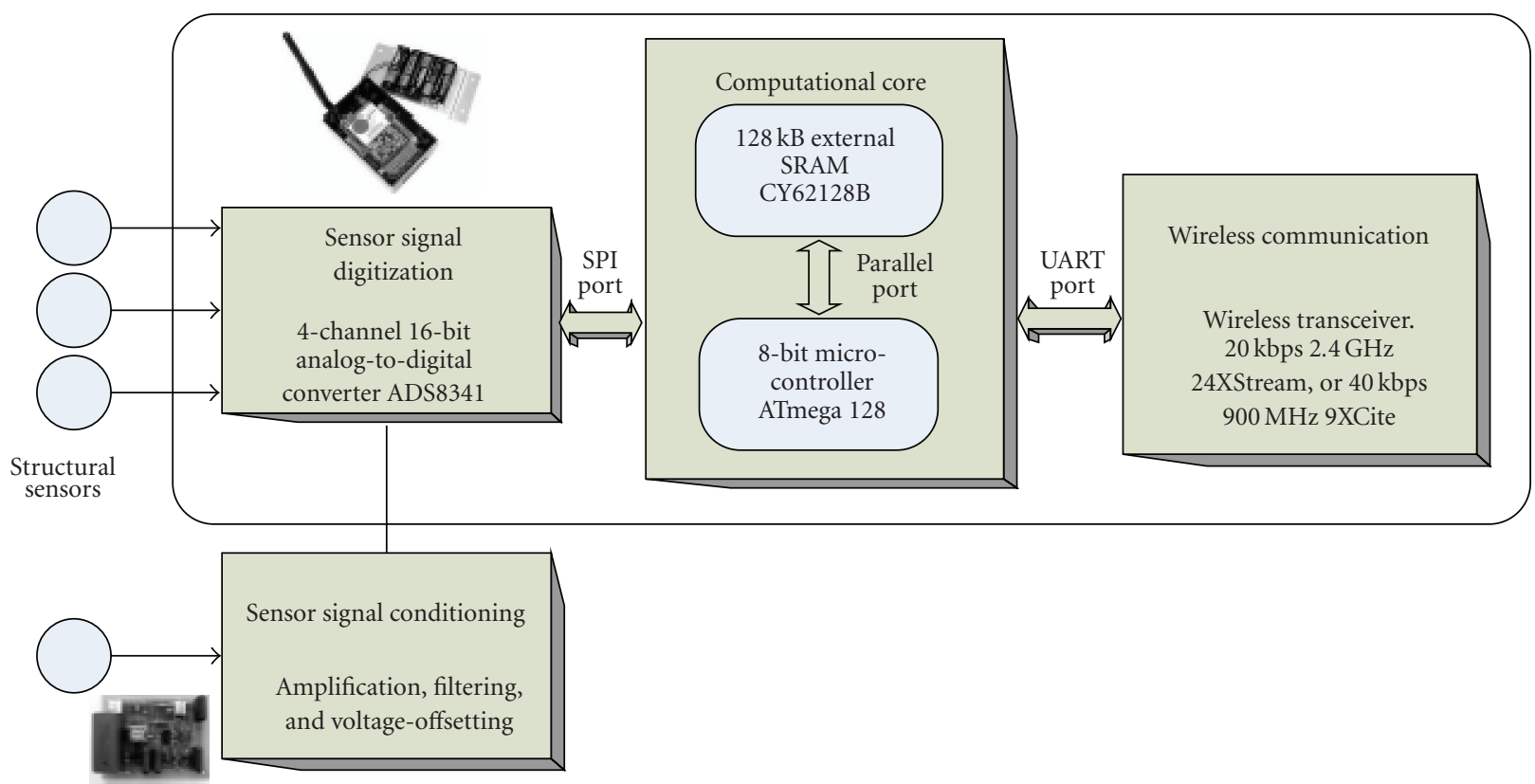

FIgURE 1: Hardware of the academic wireless sensing unit.

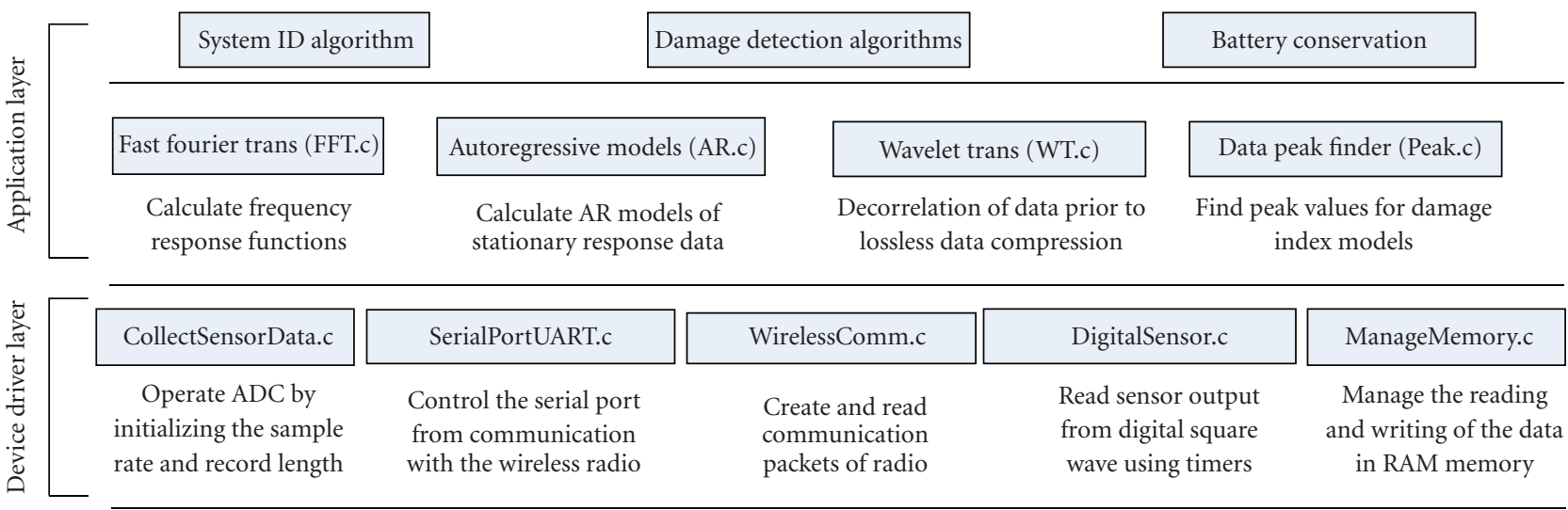

\section{Wireless sensing unit hardware}

FIGURE 2: Software Architecture of the academic wireless sensing unit.

structure has no closely spaced modes. The frequency values and the imaginary components of the FRF corresponding to the peaks are recorded by each sensing node.

(4) Each sensing node transmits the peak frequencies and the corresponding imaginary components of the FRF to the central server, where the global modal frequencies of the structure are determined. Furthermore, the mode shapes of the structure can be estimated by assembling the imaginary components of the multiple FRFs at the peaks of each sensing node:

$$
\left\{\phi_{j}\right\}=\left\{\operatorname{Im}\left[H_{1}\left(i \omega_{j}\right)\right], \operatorname{Im}\left[H_{2}\left(i \omega_{j}\right)\right], \ldots, \operatorname{Im}\left[H_{\mathrm{n}}\left(i \omega_{j}\right)\right]\right\}^{T},
$$

where $\left\{\phi_{j}\right\}$ is the $j$ th mode shape, $H_{k}\left(i \omega_{j}\right)$ is the FRF of the structure at sensor location $k, n$ is the total number of sensing nodes installed on the structure, and $\operatorname{Im}[\cdot]$ denotes the imaginary component of a complex number.

In summary, the PP algorithm is implemented in a wireless sensing network for a quick engineering estimation of modal frequency and mode shapes of a structure. However, PP analysis also has drawbacks compared with other sophisticated methods, for example, PP analysis cannot properly handle closed spaced modes [11]. To investigate the performance of the embedded FFT and PP algorithms in the wireless sensing network, laboratory and field experiments need to be conducted. 


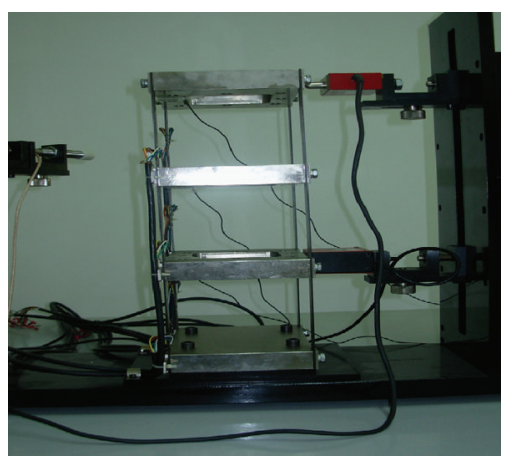

(a)

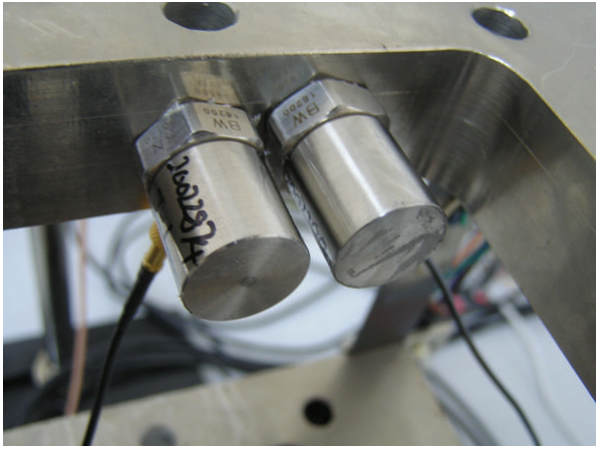

(b)

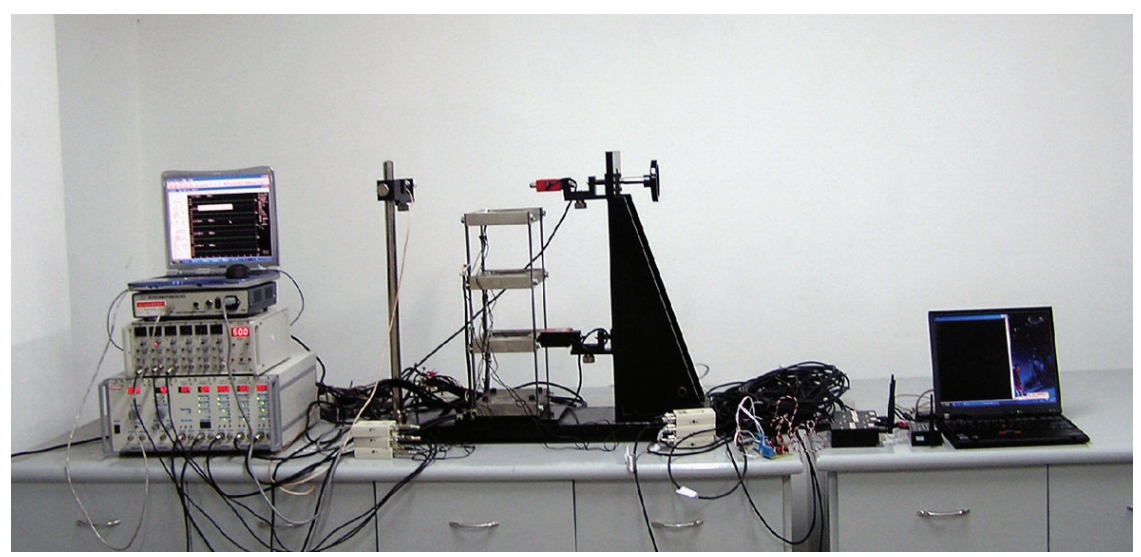

(c)

Figure 3: Test structure model with wireless sensing system.

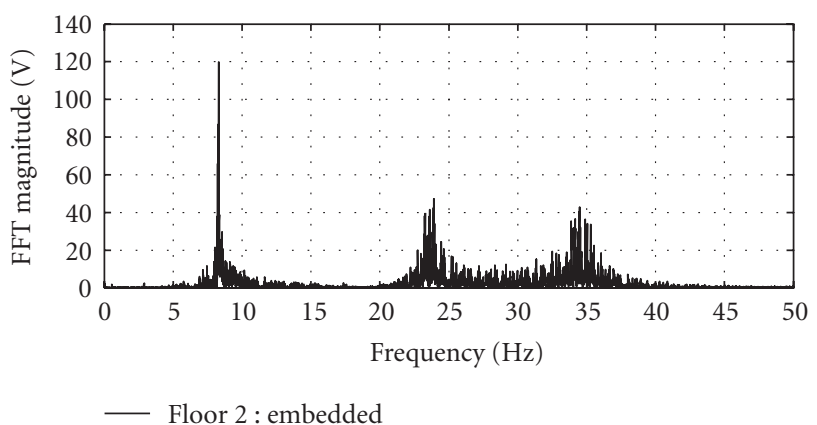

(a) Embedded FFT

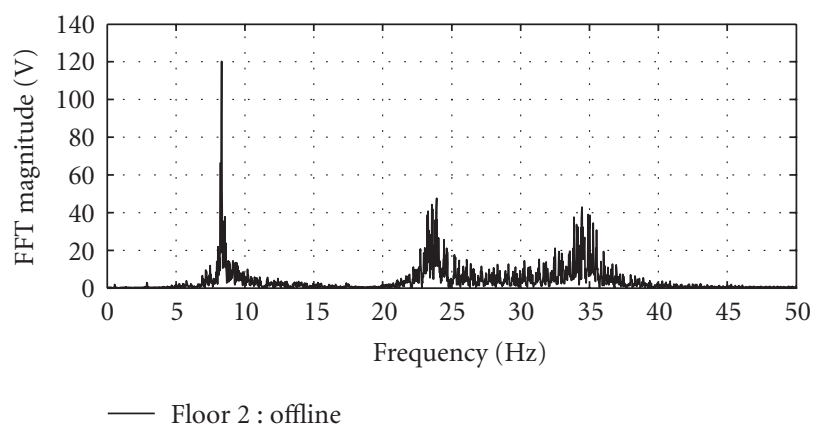

(b) Offline FFT

FIGURE 4: Comparison of FFT results. (a) Embedded using the wireless sensing unit. (b) Offline using MATLAB.

\section{Laboratory Experimental Studies}

The performance of the two algorithms embedded in the wireless sensing network is first studied upon a threestory building structure constructed in the laboratory. The structural model behaves as a lumped-mass shear building because of its rigid floors and flexible columns, as shown in Figure 3. At each floor level, light-weight BW-16200 accelerometers, manufactured by B\&W Sensing Tech, are mounted to record the acceleration response of the forced vibration of the building. The wireless sensing network with embedded algorithms is utilized for both data acquisition and processing.

First, the time-history response data is transformed to the frequency domain via the embedded FFT algorithm. Embedded computing time of the 4096-point FFT by the microcontroller is around 16.5 seconds. Based upon the datasheets of the components in the wireless sensing unit $[10,12]$, during embedded computing, the microcontroller together with the external memory consumes about only $150 \mathrm{~mW}$. On the other hand, transmission time for the 4096-point raw time-history data is around 12.6 seconds. 


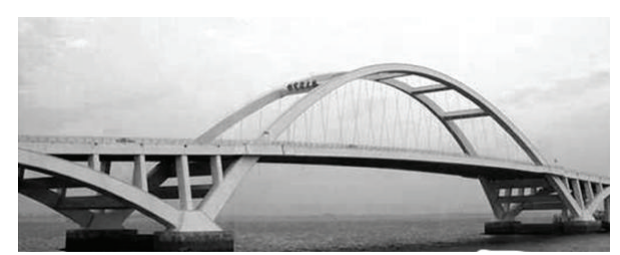

(a)

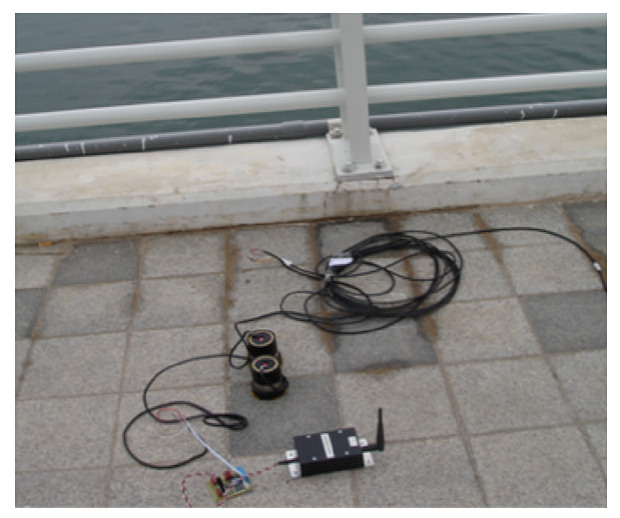

(b)

Figure 5: (a) Side view of the Wuyuan Bridge; (b) wireless sensing node on the bridge.

The total power consumption for wireless data transmission, including the necessary operations and of the microcontroller, memory units, the transceivers, and so forth, is estimated to be $900 \mathrm{~mW}$, when the $2.4 \mathrm{GHz}$ MaxStream 24XStream transceiver is adopted. Therefore, by employing the embedded FFT algorithm, battery power consumption is reduced by nearly $80 \%$.

To compare the accuracy of the embedded FFT algorithm, the frequency spectrum calculated by the embedded algorithm in the wireless sensing unit at the second floor is compared with that estimated offline by MATLAB using the same raw time-history response transmitted by the wireless sensing unit. As shown in Figure 4, the embedded FFT and offline FFT values are in close agreement.

After the validation of the embedded FFT algorithm, the embedded PP algorithm in each wireless sensing node is employed with the aforementioned procedures. Under forced vibration in the lab tests, relatively high-threshold values can be assigned for executing the embedded PP algorithm. Upon the embedded computing, each wireless sensing unit transmits the identified peak frequencies and corresponding imaginary components of the FRFs to the central data repository. These results are compared with those obtained from an offline PP analysis of the same time-history data transmitted by the three wireless sensing units. As shown in Table 1, the modal frequencies and the imaginary components of the FRFs at those frequencies estimated by embedded and offline PP analysis are identical.

The total execution time of the embedded PP algorithm (including the FFT analysis) by the ATmega128 is around 25 seconds. Then, each sensing node only transmits three peak frequencies and the corresponding imaginary components in frequency spectrum. A total of 3 (sensing nodes) $\times$ 4 (bytes per floating point number) $\times 6$ (floating point numbers) $=72$ bytes of data are transmitted by the wireless sensing network to the central server, which is much smaller compared with transmitting the 3 (sensing nodes) $\times 2$ (bytes per ADC sample) $\times 4096$ (data samples) $=24,576$ bytes of raw time-history data recorded by the three sensing nodes. According to the aforementioned power consumption of the microcontroller and the transceiver, energy saving by employing the embedded PP algorithm is again significant.

The embedded FFT and PP algorithms provide a simple approach to estimate the modal frequencies and mode shapes based upon (3). To validate the accuracy of the estimated modal frequencies and mode shapes by the two embedded algorithms, the measured time-history data, which is wirelessly transmitted to the central data repository, is processed by the stochastic subspace identification (SSI) algorithm. The SSI algorithm is an advanced time-domain system identification approach for identifying the modal frequencies and mode shapes of the structure. As shown in Table 2, the modal frequencies and mode shapes identified by embedded FFT and PP algorithms are in close agreement with those from the SSI analysis, except for minor discrepancy in the 3rd mode shape. This minor discrepancy is due to the simplicity and lower accuracy of the PP analysis. Table 2 also illustrates the Modal assurance criteria (MAC) for quantifying the similarity between identified modes.

From the lab testing results, it is shown that the embedded FFT and PP algorithms are executed in a distributed manner by individual wireless sensing nodes, which is well suited for the distributed computing paradigm of the wireless sensing network. Because the lengthy time-history vibration data do not need to be wirelessly transmitted, the decentralized computing architecture is power efficient and can significantly increase the battery life spans of the wireless sensing nodes.

\section{Field Experimental Studies on the Wuyuan Bridge}

Field experimental studies provide a more realistic way to assess the performance of the embedded FFT and PP algorithms in the wireless sensing network. A wireless sensing network is set up on the Wuyuan steel arch bridge in Xiamen, China. The bridge shown in Figure 5 is a halfthrough basket-type arch bridge. The main span is $210 \mathrm{~m}$ long with the main deck supported by steel hangers from the arched ribs. The bridge is located in Wuyuan bay, as part of the ring road around the Xiamen Island in South East China Sea.

Due to the easy installation of wireless sensors in the field, 45 locations on the bridge deck and arch ribs are mounted with uniaxial or triaxial accelerometers, manufactured by Harbin Institute of Engineering Mechanics, to measure the corresponding vertical and transverse acceleration responses of the bridge under ambient excitation (Figure 6). The uniaxial accelerometer has a sensitivity of $1 \mathrm{~V} / \mathrm{g}$ with a resolution of $10^{-6} \mathrm{~g}$, while the triaxial one has values of 


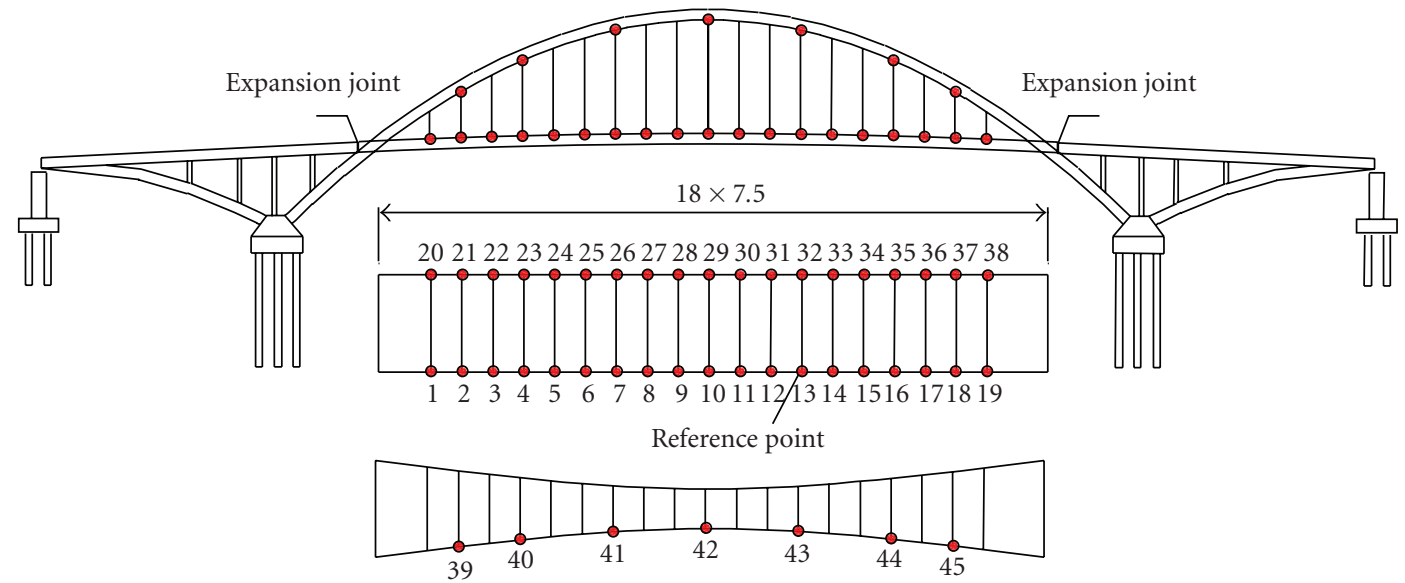

Figure 6: Locations of sensing units on the Wuyuan Bridge.

TABle 1: Comparison of the results by embedded PP and offline PP.

\begin{tabular}{lccccccccccccc}
\hline & \multicolumn{3}{c}{} & \multicolumn{4}{c}{ Embedded PP } & \multicolumn{2}{c}{} & \multicolumn{3}{c}{ Offline PP } \\
& \multicolumn{2}{c}{ 1st Peak } & \multicolumn{2}{c}{ 2nd Peak } & \multicolumn{2}{c}{ 3rd Peak } & \multicolumn{2}{c}{ 1st Peak } & \multicolumn{2}{c}{ 2nd Peak } & \multicolumn{2}{c}{ 3rd Peak } \\
& Freq. & $\operatorname{Im}(H)$ & Freq. & $\operatorname{Im}(H)$ & Freq. & $\operatorname{Im}(H)$ & Freq & $\operatorname{Im}(H)$ & Freq. & $\operatorname{Im}(H)$ & Freq. & $\operatorname{Im}(H)$ \\
\hline 1st Floor & 8.30 & -68.77 & 23.9 & -96.91 & 34.47 & 27.50 & 8.30 & -68.77 & 23.9 & -96.91 & 34.47 & 27.5 \\
2nd Floor & 8.30 & -119.8 & 23.9 & -47.26 & 34.47 & -42.8 & 8.30 & -119.8 & 23.9 & -47.26 & 34.47 & -42.8 \\
3rd Floor & 8.30 & -152.5 & 23.9 & 82.58 & 34.47 & 19.24 & 8.30 & -152.5 & 23.9 & 82.58 & 34.47 & 19.24 \\
\hline
\end{tabular}

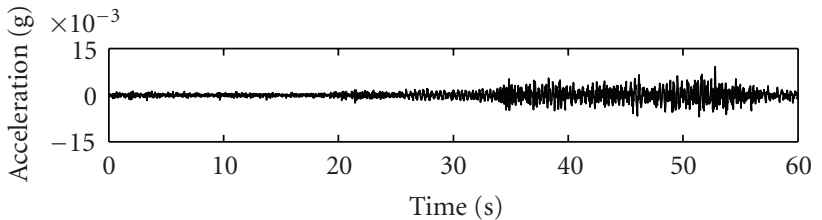

— Wireless

(a)

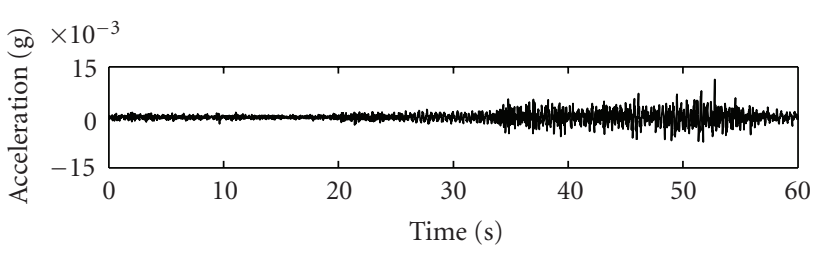

— Wired

(b)

Figure 7: Vertical acceleration time history recorded by sensing node 2 .

$8 \mathrm{~V} / \mathrm{g}$ and $1.6 \times 10^{-7} \mathrm{~g}$, respectively. For the data acquisition by the wireless sensing network, a sampling frequency of $50 \mathrm{~Hz}$ is selected. Figure 7 shows one typical vertical acceleration measurement on the bridge deck. The peak value of the recorded signal is around $0.012 \mathrm{~g}$ under normal traffic loading. A signal conditioning circuit proposed by

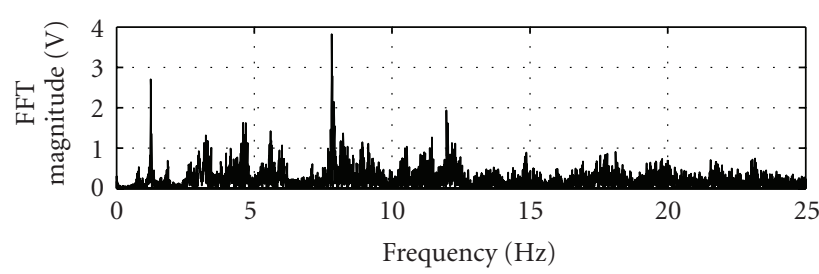

— Embedded

(a) Embedded FFFT

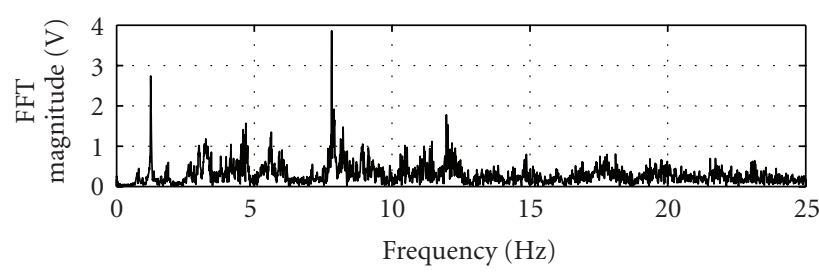

— Offline

(b) Offline FFT

Figure 8: Comparison of FFT results at sensing node 5. (a) Embedded using the wireless sensing unit. (b) Offline by MATLAB.

Lynch et al. [7] and Wang et al. [8] is included in each sensing node to both amplify and band-pass the analog accelerometer signal, which is especially useful for recording weak ambient vibration. Since this paper focuses on studying the performance of the embedded FFT and PP algorithm in the field experiments on the Wuyuan Bridge, more detailed 


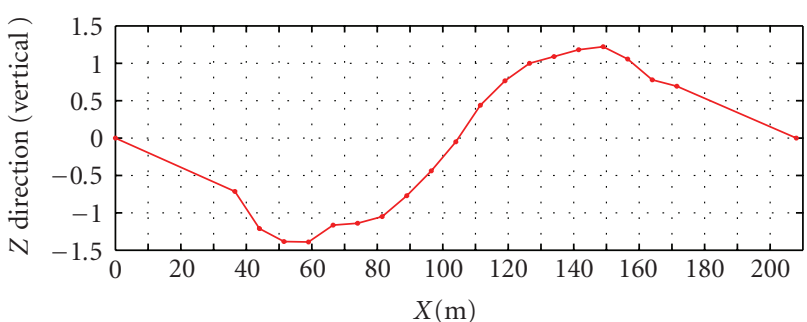

(a) The 1st mode

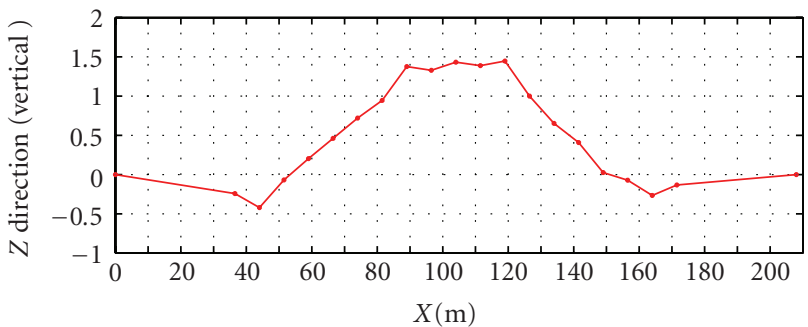

(b) The 2nd mode

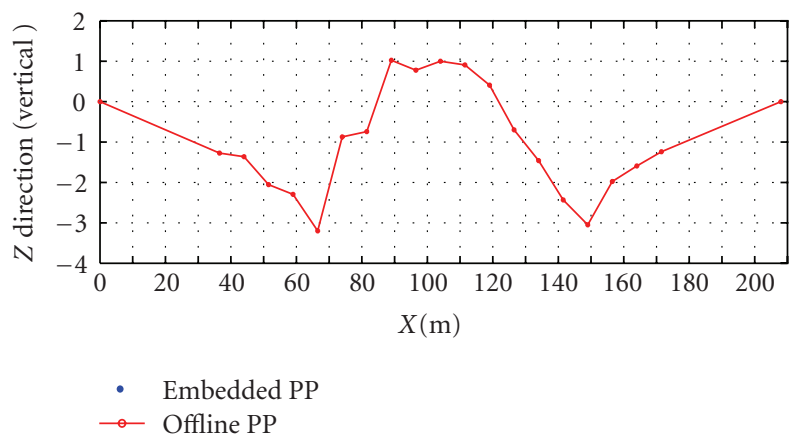

(c) The 3rd mode

Figure 9: Comparison of mode shapes estimated by embedded and offline PP.

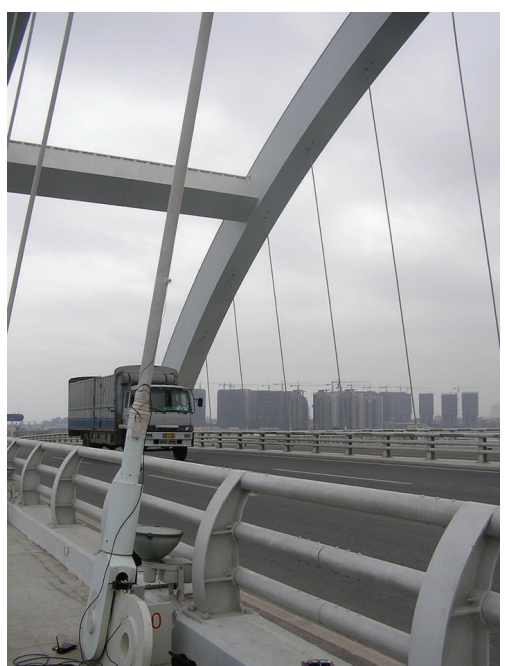

(a)

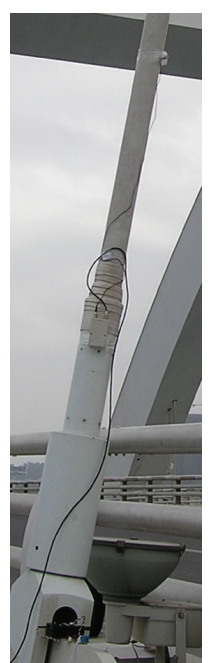

(b)
Figure 10: Accelerometer with wireless sensor on a hanger.

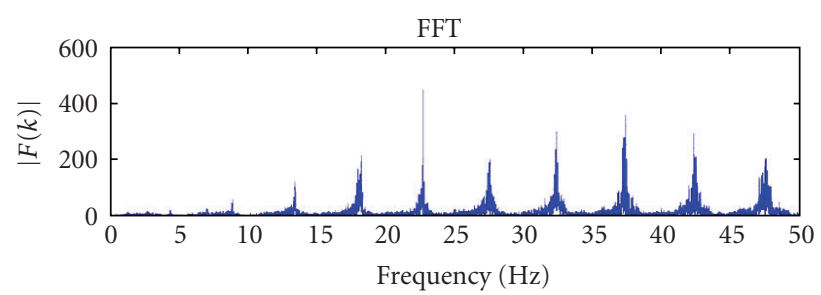

FIGURE 11: Frequency spectrum of the vibration of a hanger.

TABle 2: Comparison of identified modal frequencies and mode shapes.

\begin{tabular}{lcccccc}
\hline & \multicolumn{2}{c}{ Mode 1 } & \multicolumn{2}{c}{ Mode 2 } & \multicolumn{2}{c}{ Mode 3 } \\
& PP & SSI & PP & SSI & PP & SSI \\
\hline $\begin{array}{l}\text { Frequency (Hz) } \\
\text { 1st Floor Modal }\end{array}$ & 8.30 & 8.31 & 23.90 & 23.59 & 34.47 & 34.54 \\
$\begin{array}{l}\text { Component } \\
\text { 2nd Floor }\end{array}$ & 0.451 & 0.450 & -1.174 & -1.177 & 1.429 & 1.839 \\
$\begin{array}{l}\text { Modal } \\
\text { Component }\end{array}$ & 0.786 & 0.786 & -0.572 & -0.545 & -2.224 & -2.24 \\
$\begin{array}{l}\text { 3rd Floor Modal } \\
\text { Component }\end{array}$ & 1.000 & 1.000 & 1.000 & 1.000 & 1.000 & 1.000 \\
\hline MAC & 1.000 & \multicolumn{2}{c}{0.998} & 0.978 \\
\hline
\end{tabular}

TABLE 3: Identified vibration frequencies of the Wuyuan Bridge in vertical bending by the embedded and offline PP analysis.

\begin{tabular}{lccc}
\hline Frequencies Embedded PP & Offline PP & Mode Shape Description \\
\hline 1 & 0.778 & 0.775 & 1st Vertical Bending \\
2 & 1.24 & 1.24 & 2nd Vertical Bending \\
3 & 1.70 & 1.70 & 3rd Vertical Bending \\
\hline
\end{tabular}

TABLE 4: Comparisons of the identified frequencies of the cable by embedded PP and offline SSI.

\begin{tabular}{lcc}
\hline Frequencies & Embedded PP & Offline SSI \\
\hline 1st & $/$ & $/$ \\
2nd & $/$ & 8.985 \\
3rd & 13.50 & 13.47 \\
4th & 18.28 & 18.19 \\
5th & 22.76 & 22.73 \\
6th & 27.44 & 27.50 \\
7th & 32.28 & 32.34 \\
8th & 37.21 & 37.30 \\
\hline
\end{tabular}

information about the field experiments with the wireless sensing network can be found in Lei et al. [9]. First, the time-history response data collected by the sensing nodes is transformed to the frequency domain via the embedded FFT algorithm. As the sampling frequency is $50 \mathrm{~Hz}$ and there are 4096 points in the FFT data, the frequency resolution can be estimated as

$$
f_{r}=\frac{f_{\mathrm{Nyquist}}}{N / 2}=\frac{25}{2048}=0.012 \mathrm{~Hz}
$$


For validating the accuracy of the embedded FFT algorithm, the raw time history of the acceleration response data collected by the sensing node is also transmitted to the central server and transformed to frequency domain by MATLAB. As shown in Figure 8, the embedded FFT and offline FFT spectra are in close agreement.

Then, the embedded PP algorithm employed to process each vertical acceleration response recorded by the sensing node 1-19 in Figure 6. Under ambient vibration condition in the field, relatively low-threshold values with $\beta=2 \mu$ are assigned in the PP analysis, in order to avoid missing small peaks in the frequency spectra. The total execution time of the embedded PP algorithm (including FFT analysis) by each ATmega 128 microcontroller is still around 25 seconds. Each sensing node transmits the identified peak frequencies and corresponding imaginary components of the FRFs to the central data repository, where global modal frequencies and mode shapes of the bridge in the vertical direction are determined.

To validate the accuracy of the embedded PP algorithm, PP analyses of the same time-history data transmitted by the 19 sensing nodes are also conducted in MATLAB. Table 3 lists the first three vertical bending frequencies of the bridge. The frequencies estimated by the embedded PP algorithm are shown in the second column, and the frequencies estimated by offline PP analysis are shown in the third column. The two sets of results are in close agreement. In addition, Figure 9 shows the corresponding first three vertical bending mode shapes, including the results estimated by both the embedded and offline PP analysis. The two sets of mode shapes are also in close agreement. The errors in the identified mode shapes are due to the fact that PP is a simple and low-accuracy approach for modal analysis.

For identifying the first three vertical modes of the bridge by the embedded FFT and PP algorithms, only 19 (sensing nodes) $\times 4$ (bytes per floating point number) $\times 6$ (floating point numbers) $=456$ bytes of the analysis results are transmitted by the 19 wireless sensing nodes to the central data repository. However, for offline identification of the modal properties, a total of 19 (sensing nodes) $\times 2$ (bytes per ADC sample) $\times 4096$ (data samples) $=155,648$ bytes of data need to be wirelessly transmitted. Obviously, employing embedded FFT and PP algorithms can greatly reduce data transmission and energy consumption for a large-scale wireless sensing network deployed in the field.

Bridge cable forces are important parameters for the assessment of bridge safety. Some techniques, including utilizing wireless sensing, have been developed for the measurement of steel bridge cable forces [13-15]. For bridges in service, steel cable forces are usually monitored based upon the vibration frequencies of cables [16]. Through the analysis of the transverse vibration of a steel cable, force $T$ in a steel cable can be approximated by [16]

$$
T=4 m l^{2} \frac{f_{k}^{2}}{k^{2}}
$$

in which $l$ and $m$ are the length and the mass per unit length of the cable, respectively, and $f_{k}$ is the $k$ th modal frequency of the cable. Therefore, cable forces can be estimated once the vibration frequencies of a cable are identified. Due to the efficient computing approach offered by the embedded FFT and PP algorithms in the wireless sensing unit, vibration frequencies of a cable can be identified in situ by the wireless unit. An accelerometer is attached to a hanger of the bridge to measure the vibration of the cable and a wireless sensing unit with embedded FFT and PP algorithm is used for data acquisition and processing, as shown in Figure 10.

Only the peak vibration frequencies identified by the embedded PP algorithm are transmitted to the central server. The results are shown in the second column of Table 4. Although a small threshold value is selected in executing the embedded PP algorithm, the amplitudes of the spectrum peaks at the first two frequencies are still too small to be identified, because the accelerometer is attached very close to the end of the hanger. However, since focus is now upon the estimation of the cable forces utilizing (4), which does not require all the peaks in the frequency spectrum, the first and the second vibration frequencies of the cable are not necessary in this study.

To validate the accuracy of the frequencies identified by the embedded PP algorithm, the recorded time-history data by the sensing unit are wirelessly transmitted to the central data repository, where the sophisticated SSI algorithm is applied to process the data. The identified frequencies are shown in the third column of Table 4 . From the side-by-side comparison, it is evident that the embedded PP algorithm can pick up the vibration frequencies of the cable accurately. Based upon the identified vibration frequencies, the force in the cable can be estimated using the frequency method as indicated in (4).

\section{Conclusions}

In this paper, the performance of two engineering algorithms, the fast Fourier transform (FFT) and peak-picking (PP) algorithms, embedded in an academic wireless sensor prototype is investigated through laboratory and field experimental studies. Comparison with the results obtained by corresponding offline FFT and PP analyses of the transmitted time-history data validates the effectiveness of the two embedded algorithms. It is shown that the frequency spectra calculated by the embedded FFT are accurate in both the laboratory and field studies. The embedded PP algorithm provides a simple and efficient approach for estimating the modal frequencies and mode shapes of a structure. The results can also be used to estimate forces in bridge cables. Since self-interrogation of measurement data by the two embedded algorithms greatly reduces the amount of data to be transmitted by the wireless sensor network, intelligent wireless sensing with embedded algorithms offer network solutions that are both scalable and power efficient.

In this paper, emphasis is placed on the implementation and performance of the two engineering algorithms in the wireless sensor system for modal identification through lab and field investigation. The wireless sensor system was installed on the lab structure or on the bridge only for shortterm operation, to compare the identification results by the embedded algorithms with those obtained by conventional 
offline analyses. Long-term monitoring utilizing the wireless sensor system and performance of the system, including the efficiency of the embedded algorithms in various climatic conditions, were not studied in this work. It will be important to fully investigate the robustness and lifespan of the proposed algorithm and system before they can serve as a substitute for conventional wire-based monitoring system, particularly for long-term monitoring of engineering structures. Such studies will be conducted by the authors in the future.

\section{Acknowledgments}

This paper is partially supported by China National HighTech Research and Development Program through Grant no. 2007AA04Z420. The first author also acknowledges support from the program for New Century Excellent Talents in Universities (NCET) from China National Education Ministry.

\section{References}

[1] B. F. Spencer Jr., M. E. Ruiz-Sandoval, and N. Kurata, "Smart sensing technology: opportunities and challenges," Structural Control and Health Monitoring, vol. 11, no. 4, pp. 349-368, 2004.

[2] J. P. Lynch and K. J. Loh, "A summary review of wireless sensors and sensor networks for structural health monitoring," Shock and Vibration Digest, vol. 38, no. 2, pp. 91-128, 2005.

[3] Y. Wang, Wireless sensing and decentralized control for civil structures: theory and implementation, Ph.D. thesis, Department of Civil and Environmental Engineering, Stanford University, Stanford, Calif, USA, 2007.

[4] J. P. Lynch, A. Sundararajan, K. H. Law, A. S. Kiremidjian, T. Kenny, and E. Carryer, "Embedment of structural monitoring algorithms in a wireless sensing unit," Structural Engineering and Mechanics, vol. 15, no. 3, pp. 285-297, 2003.

[5] J. P. Lynch, "An overview of wireless structural health monitoring for civil structures," Philosophical Transactions of the Royal Society A, vol. 365, no. 1851, pp. 345-372, 2007.

[6] S. D. Glaser, M. Li, M. L. Wang, J. Ou, and J. Lynch, "Sensor technology innovation for the advancement of structural health monitoring: a strategic program of US-China research for the next decade," Smart Structures and Systems, vol. 3, no. 2, pp. 221-244, 2007.

[7] J. P. Lynch, Y. Wang, K. J. Loh, J.-H. Yi, and C.-B. Yun, "Performance monitoring of the Geumdang bridge using a dense network of high-resolution wireless sensors," Smart Materials and Structures, vol. 15, no. 6, pp. 1561-1575, 2006.

[8] Y. Wang, K. Loh, J. P. Lynch, M. Fraser, K. H. Law, and A. Elgamal, "Vibration monitoring of the Voigt bridge using wired and wireless monitoring systems," in Proceedings of the 4th China-US-Japan Symposium on Structural Control and Monitoring, Hangzhou, China, October 2006.

[9] Y. Lei, W. A. Shen, Y. Song, and J. P. Lynch, "Application of wireless monitoring system for the ambient vibration study of the Wuyuan steel arch bridge," in Proceedings of the 1st World Forum on Smart Materials and Smart Structures Technology (SMSST '07), Chongqing \& Nanjing, China, 2007.

[10] Y. Wang, J. P. Lunch, and K. W. Law, "Information driven wireless sensing and control for civil structures," in Proceedings of the 1st World Forum on Smart Materials and Smart Structures Technology (SMSST '07), Chongqing \& Nanjing, China, 2007.

[11] A. T. Zimmerman, M. Shiraishi, R. A. Swartz, and J. P. Lynch, "Automated modal parameter estimation by parallel processing within wireless monitoring systems," Journal of Infrastructure Systems, vol. 14, no. 1, pp. 102-113, 2008.

[12] MaxStream, Inc., "XStream ${ }^{\mathrm{TM}}$ OEM RF Module Product Manual v4.2B," Lindon, Utah, USA, 2005.

[13] G. Feltrin, J. Meyer, R. Bischoff, and M. Motavalli, "Wireless sensor networks for long term monitoring of civil structures," Journal of Structure \& Infrastructure Engineering, pp. 1-14, 2009.

[14] G. Feltrin, J. Meyer, and R. Bischoff, "A wireless sensor network for force monitoring of cable stays," in Proceedings of the 3rd International Conference on Bridge Maintenance, Safety and Management (IABMAS '06), Porto, Portugal, 2006.

[15] S. Cho, C. B. Yun, J. P. Lynch, A. T. Zimmerman, B. F. Spencer Jr., and T. Nagayama, "Smart wireless sensor technology for structural health monitoring of civil structures," Journal of Steel Structures, vol. 8, pp. 267-275, 2008.

[16] J. C. Russell and T. J. Lardner, "Experimental determination of frequencies and tension for elastic cables," Journal of Engineering Mechanics, vol. 124, no. 10, pp. 1067-1072, 1998. 

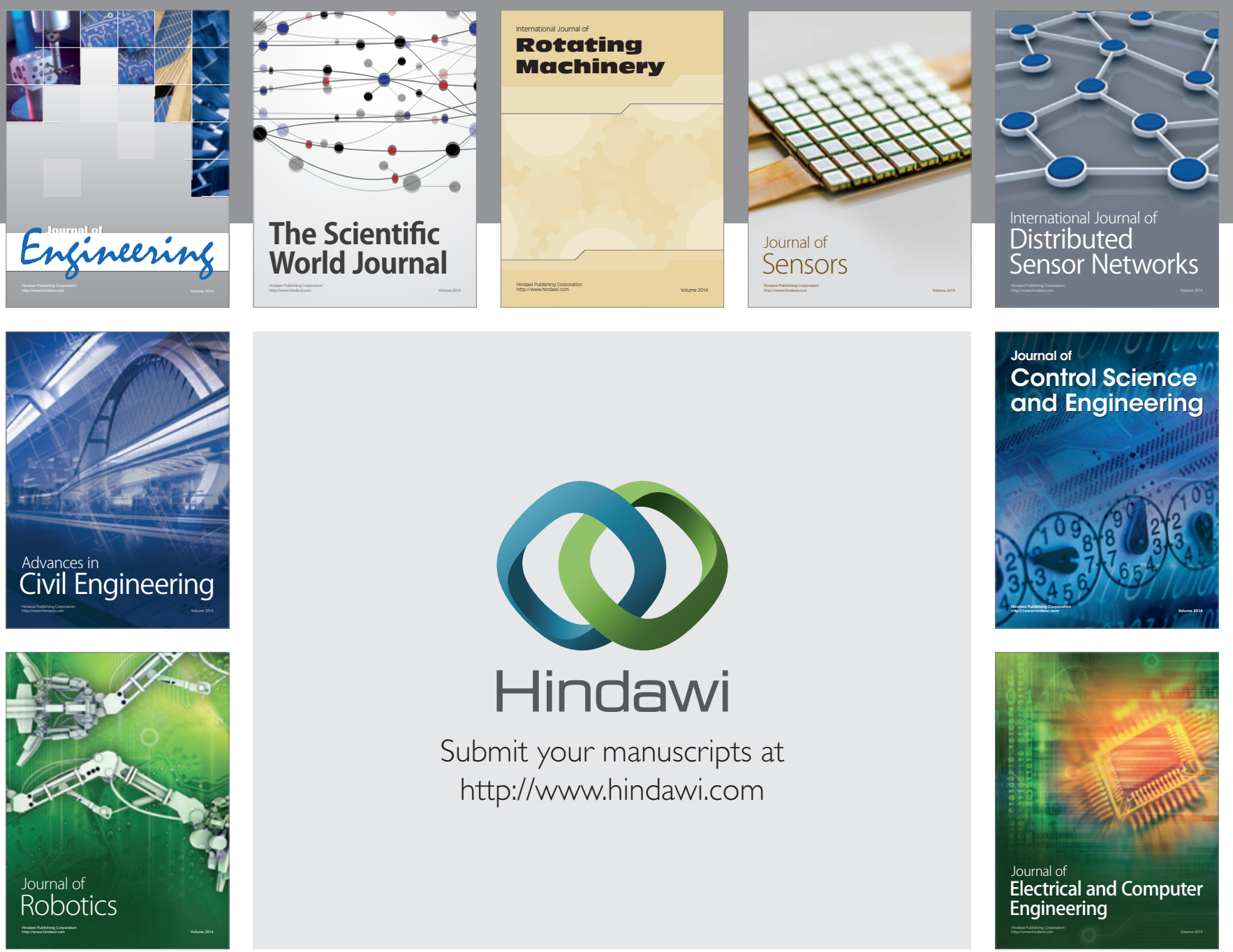

Submit your manuscripts at

http://www.hindawi.com
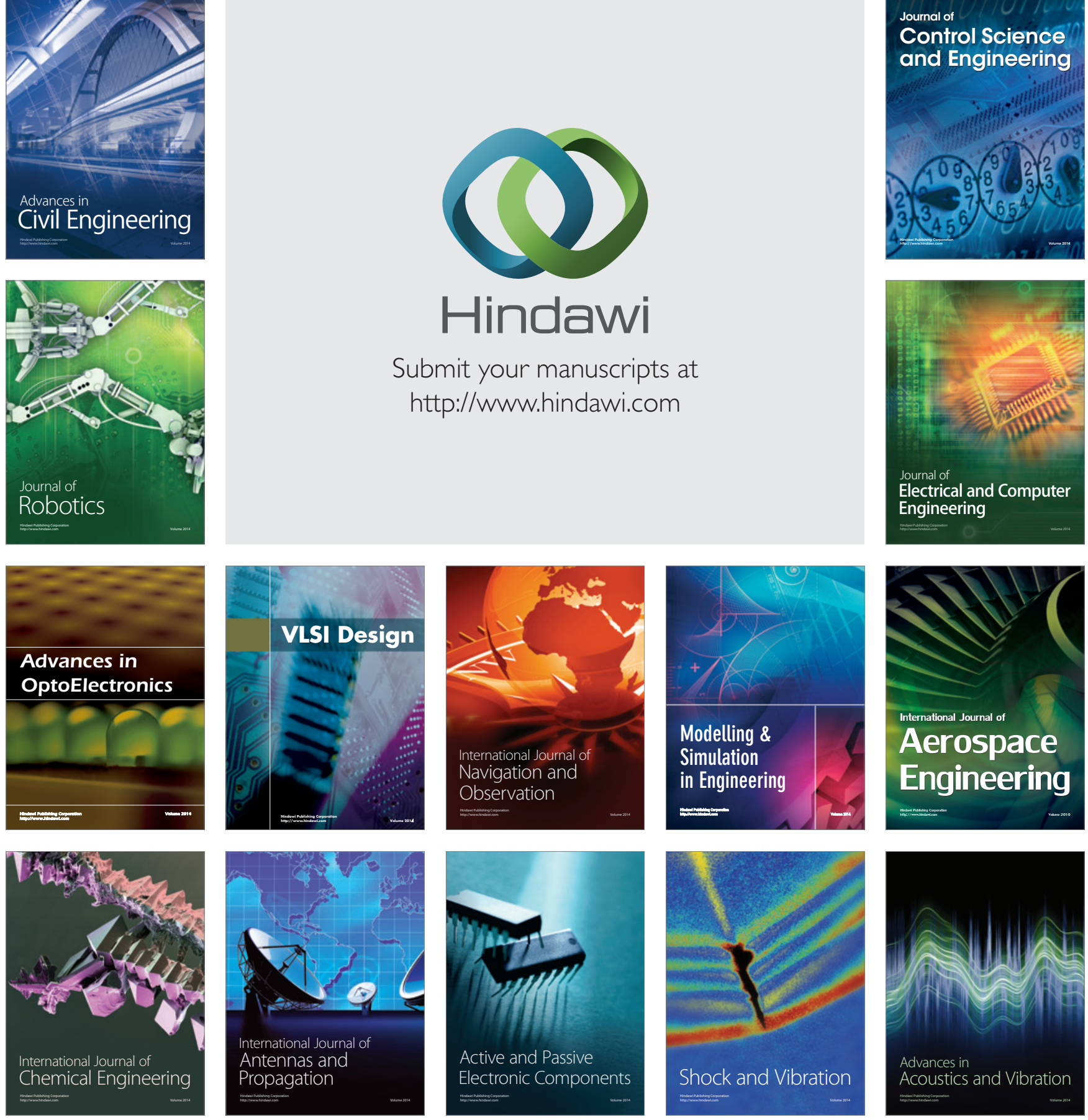